\title{
Editorial
}

\section{Infosaludlaboral, un nuevo portal de acceso a la información científica en salud y seguridad en el trabajo}

Desde el pasado mes de septiembre, la Escuela Nacional de Medicina del Trabajo del Instituto de Salud Carlos III, ha puesto al servicio de los diferentes agentes y actores que intervienen en progreso de la salud en el trabajo, una nueva herramienta que pretende contribuir a facilitar el acceso a la producción científico- técnica en materia de Salud y Seguridad en el Trabajo: http://Infosaludlaboral.isciii.es.

Si hay un hecho destacable en la evolución de la prevención de riesgos laborales en nuestro país, principalmente durante la primera del siglo XXI, ha sido la valorización del conocimiento como estrategia necesaria para el transito de una prevención normativa basada en la legislación hacia un prevención de excelencia de base científica.

Este hecho es convergente con el escenario marcado por el Consejo Europeo extraordinario celebrado en Lisboa en marzo de 2000: "hacia la Europa de la innovación y el conocimiento", escenario al que no puede ser ajena la seguridad y salud en el trabajo y que queda reflejado en las últimas convocatorias de ayudas a la investigación realizadas tanto por el Instituto de Salud Carlos III (ISCIII), a través de la Subdirección General de Evaluación y Fomento de la Investigación, más conocida como Fondo de Investigación Sanitaria (FIS), como por el Instituto Nacional de Seguridad e Higiene en el Trabajo (INSHT).

De esta forma, a través del marco que ofrecen los Programas de Fomento de la Investigación Biomédica y de Investigación en Evaluación de Tecnologías Sanitarias y Sistemas de Salud, por parte del Instituto de Salud Carlos III, del Programa de Investigación del Instituto Nacional de Seguridad e Higiene en el Trabajo y de otros programas desarrollados por algunas Comunidades Autónomas, queda abierto un campo de oportunidades para la investigación en prevención de riesgos laborales, que ha sido recogido en la propia Estrategia Española de Seguridad y Salud en el Trabajo 2007-2012.

Pero el aprovechamiento de estas oportunidades requiere que las propuestas investigadoras sean productivas dentro del marco de investigación competitiva y que sus resultados movilicen el conocimiento aprovechando el importante desarrollo de las Tecnologías de la Información y Comunicación (TIC) con el que podemos contar actualmente. Así, es importante que generemos herramientas que permitan acercar las fuentes de información a la comunidad científica y a los profesionales de la salud, facilitado la proliferación y difusión de la información científica accesible electrónicamente para que el conocimiento pueda trascender hacia el profesional de la prevención. 
Precisamente, este es el reto que hemos querido abordar mediante la puesta en marcha de infosaludlaboral, como un recurso científico que juegue un papel activo en el acceso a la información, facilitando el proceso de mejora de las condiciones de trabajo y de la salud de los trabajadores.

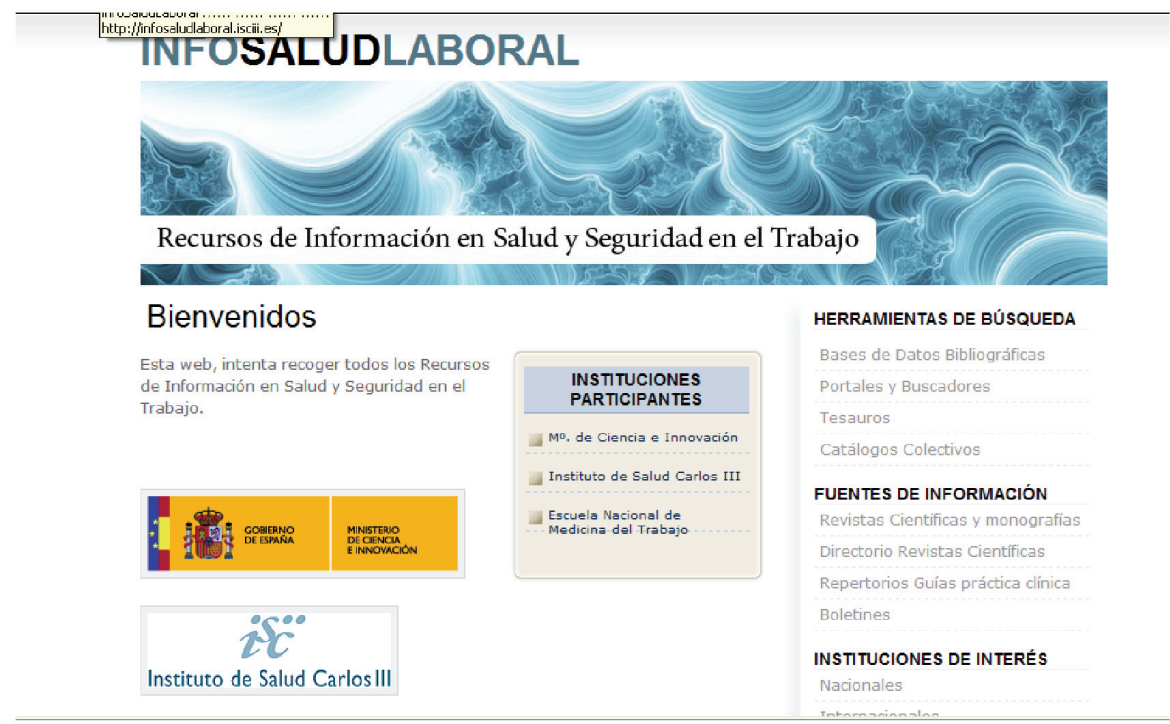

Uno de los principales valores añadidos que ofrece infosaludlaboral, reside en su simplicidad, facilitando localizar información sin que se requiera poseer conocimientos exhaustivos sobre sistemas de búsqueda de información y documentación científica. Así, a través de sus cuatro bloques principales (Herramientas de búsqueda, Fuentes de información, Instituciones de interés y Programas de financiación), permite el acceso a un total de 370 diferentes fuentes de información científico- técnica relacionadas con la salud laboral y seguridad en el trabajo.

A través del bloque de Herramientas de búsqueda, se puede acceder a 35 recursos, 15 de ellos, bases de datos bibliográficas (CISDOC, IBSST, TOXNET, WHOLIS, MEDLINE, COCHRANE-PLUS, IBECS, EXCELENCIA CIENTÍFICA, etc.), 16 buscadores y portales de información (BUSCATOX, THE INTERNET PUBLIC LIBRARY, LIS, RED-BVS, etc.), 3 tesauros (CIS, MeSH y DeCS) y 2 catálogos colectivos (C17 y SECS).

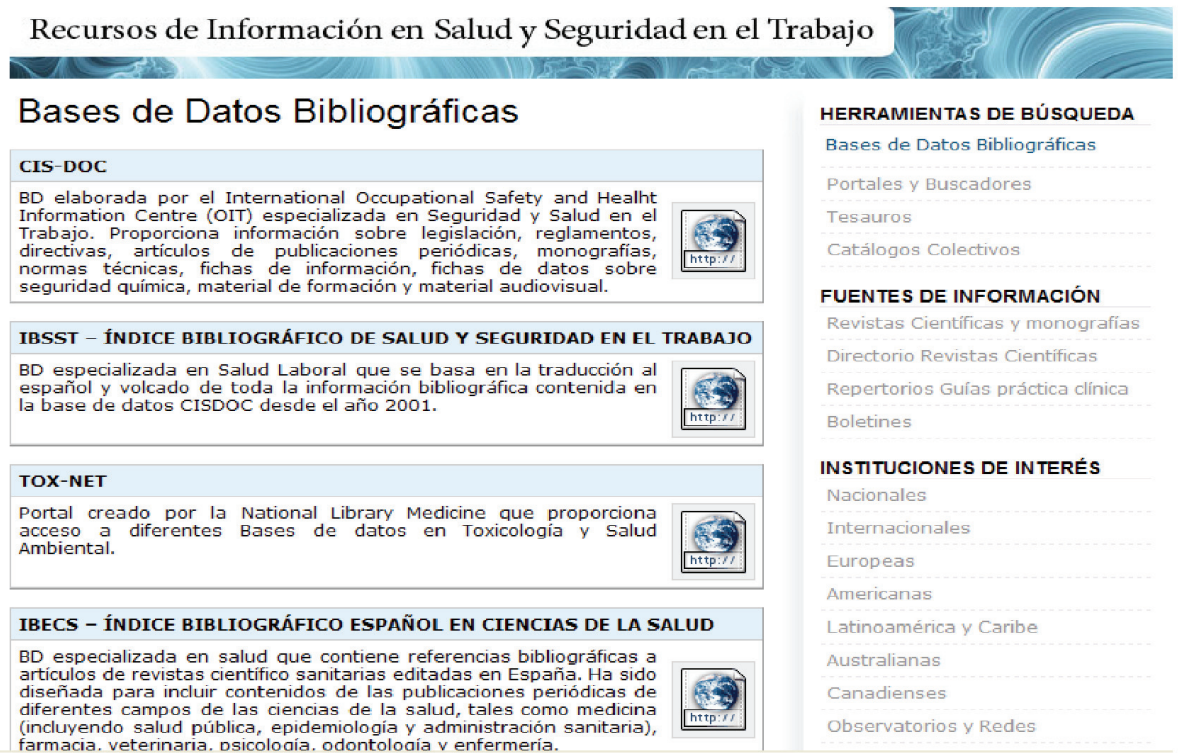


Dentro del bloque de Fuentes de información, se encuentran accesibles 61 recursos, entre ellos 13 accesos a revistas científicas y monografías a texto completo, un directorio con 46 cabeceras que ofrece información sobre revistas científicas disponibles electrónicamente y 2 repertorios de guías de práctica clínica (GUIDELINE FINDER y NCG).

\begin{tabular}{|c|c|c|}
\hline \multirow{2}{*}{\multicolumn{2}{|c|}{$\begin{array}{l}\text { Revistas Científicas y Monografías con } \\
\text { accesso a texto completo }\end{array}$}} & \multirow{3}{*}{$\begin{array}{l}\text { HERRAMIENTAS DE BÚSQUEDA } \\
\text { Bases de Datos Bibliográficas } \\
\text { Portales y Buscadores }\end{array}$} \\
\hline & & \\
\hline \multicolumn{2}{|l|}{ MONOGRAFÍAS (ACCESO LIBRE) } & \\
\hline \multirow{3}{*}{$\begin{array}{l}\text { Salud y Condiciones de Trabajo en el Transporte de Mercancias por } \\
\text { Carretera. }\end{array}$} & & Tesauros \\
\hline & & Catálogos Colectivos \\
\hline & $\Delta=$ & FUENTES DE INFORMACIÓN \\
\hline \multirow{3}{*}{$\begin{array}{l}\text { Guía de Valoración de Riesgos Laborales en el embarazo y lactancia } \\
\text { en trabajadoras del ámbito sanitario. }\end{array}$} & & Revistas Cientificas y monografias \\
\hline & & Directorio Revistas Cientificas \\
\hline & & Repertorios Guías práctica clínica \\
\hline \multirow{2}{*}{$\begin{array}{l}\text { Notas explicativas de ayuda al diagnóstico de las enfermedades } \\
\text { profesionales. }\end{array}$} & $=$ & Boletines \\
\hline & $\stackrel{\bar{\vdots}}{\square}$ & INSTITUCIONES DE INTERÉS \\
\hline \multirow{4}{*}{$\begin{array}{l}\text { Se edita por primera vez la Enciclopedia de Salud y Seguridad en el } \\
\text { Trabajo de la OIT en soporte electrónico y en lengua española. Se } \\
\text { presenta así la información contenida en ella en línea con la tecnología } \\
\text { más actual. Es de acceso gratuito desde Internet, lo que asegura su } \\
\text { difusión para todo el público interesado en la materia sobre } \\
\text { Prevención de Riesgos Laborales. }\end{array}$} & & Nacionales \\
\hline & 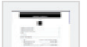 & Internacionales \\
\hline & & Europeas \\
\hline & & Americanas \\
\hline
\end{tabular}

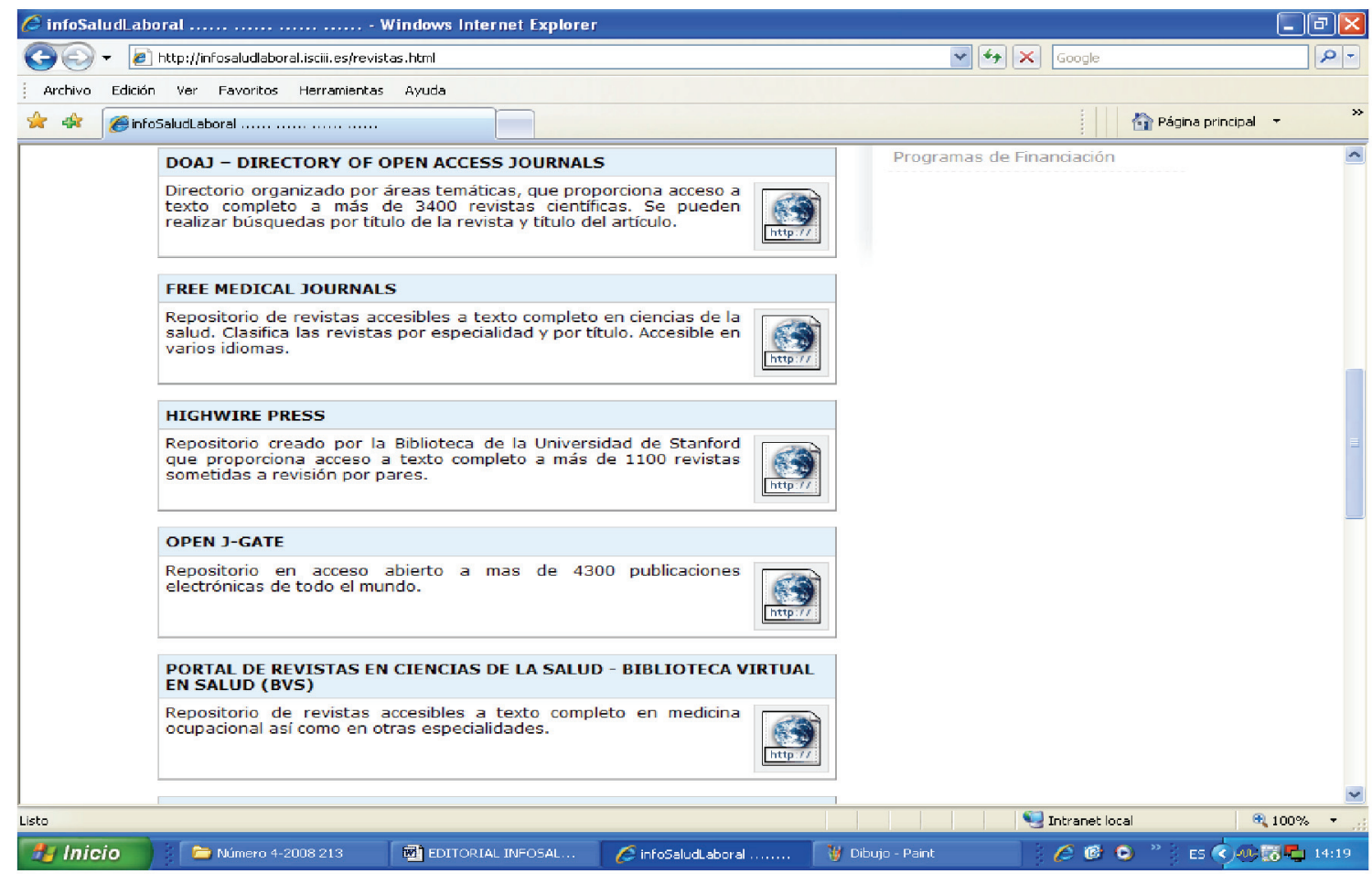


El bloque de Instituciones de interés, permite el acceso a más de 260 URL instituciones de ámbito nacional, europeo y otros ámbitos internacionales, así como a 10 observatorios o redes de seguridad y salud en el trabajo (OSL, OECT; ENWHP, etc.).

Por último, dispone de un bloque de Programas de financiación que facilita información sobre las diferentes convocatorias de ayudas a la investigación (FECYT, ISCIII, INSHT, etc.).

\section{INFOSALUDLABORAL}

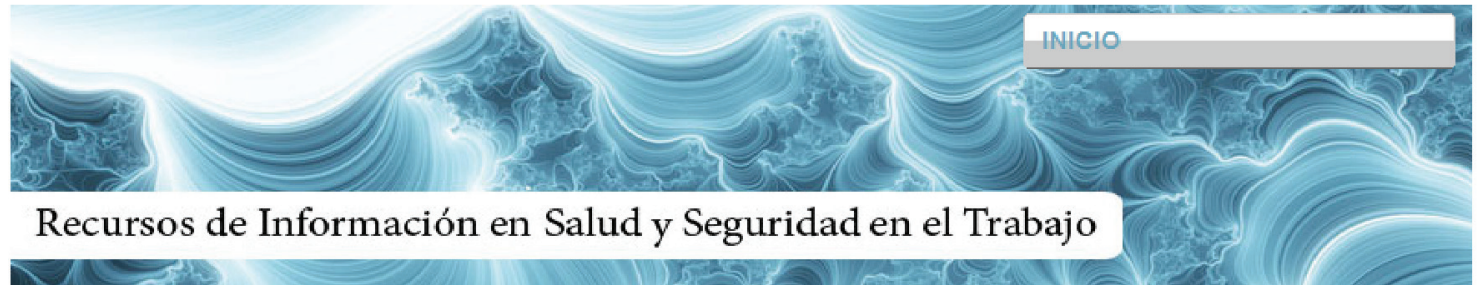

Instituciones de interés - Nacionales

\begin{tabular}{|l|}
\hline Ministerios \\
\hline Comunidades Autónomas \\
\hline Institutos \\
\hline Mutuas de Accidentes de Trabajo y Enfermedades Profesionales \\
\hline Fundaciones y Asociaciones Profesionales \\
\hline Agentes Sociales
\end{tabular}

Ultima actualización: 12/09/2008

\section{HERRAMIENTAS DE BÚSQUEDA}

Bases de Datos Bibliográficas

Portales y Buscadores

Tesauros

Catálogos Colectivos

\section{FUENTES DE INFORMACIÓN}

Revistas Científicas y monografias Directorio Revistas Cientificas

Repertorios Guías práctica clínica Boletines

INSTITUCIONES DE INTERÉS Nacionales

Recursos de Información en Salud y Seguridad en el Trabajo

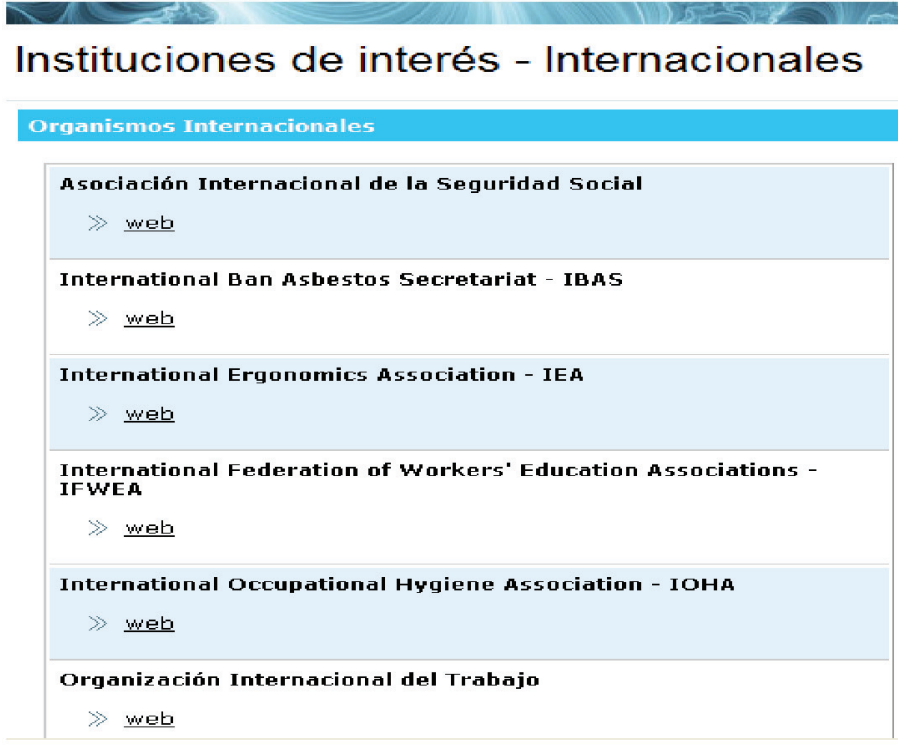

HERRAMIENTAS DE BÚSQUEDA

Bases de Datos Bibliográficas

Portales y Buscadores

Tesauros

Catálogos Colectivas

\section{FUENTES DE INFORMACIÓN}

Revistas Cientificas y monografias

Directorio Revistas Cientificas

Repertorios Guías práctica clínica

Boletines

\section{INSTITUCIONES DE INTERÉS}

Nacionales

Internacionales

Europeas

Americanas

Latinoamérica y caribe

Australianas

Canadienses

Observatorios y Redes 


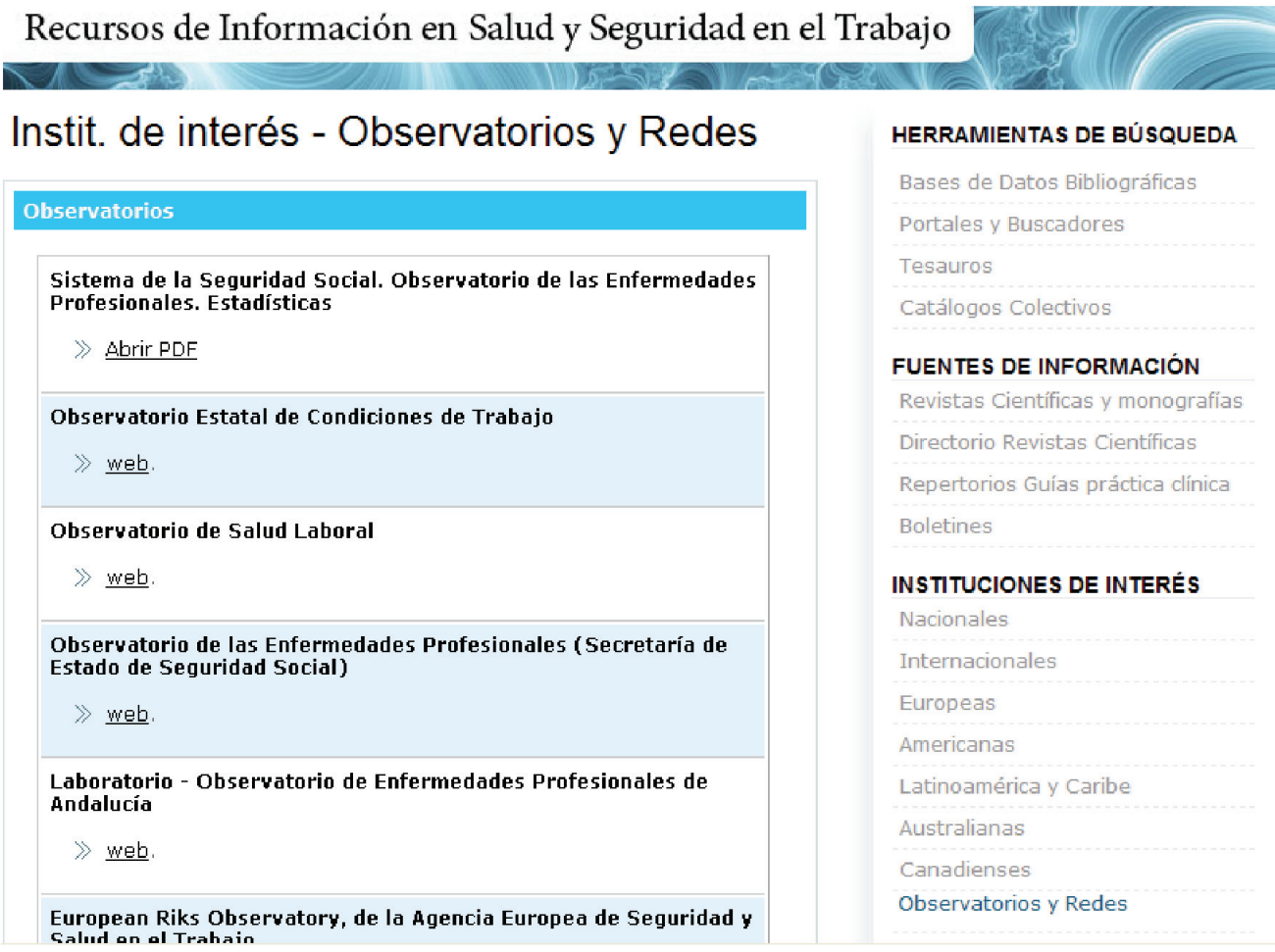

Con esta iniciativa, que se enmarca dentro de la misión que la Escuela Nacional de Medicina del Trabajo asume en cuanto a promoción del conocimiento científico sobre la enfermedad profesional y, en general, de la enfermedad ligada a las condiciones de trabajo, hemos querido contribuir a fomentar la visibilidad de la información como germen de ciencia y como herramienta de ayuda para resolver problemas de la práctica diaria dentro del variado espectro que constituye la Sociedad del Trabajo Española.

Jerónimo Maqueda Blasco

Jorge Veiga de Cabo

Escuela Nacional de Medicina del Trabajo, Instituto de Salud Carlos III, Madrid; España 